\title{
Assessment of the Therapeutic Effect of Total Glucosides of Peony for Juvenile Idiopathic Arthritis: A Systematic Review and Meta-Analysis
}

\author{
Yongsong Cai, ${ }^{1}$ Qiling Yuan, ${ }^{1} \mathrm{Ke} \mathrm{Xu},{ }^{2}$ Jialin $\mathrm{Zhu},{ }^{2}$ Yuanbo Li, ${ }^{2}$ \\ Xiaoqing $\mathrm{Wu},{ }^{2}$ Le Yang, ${ }^{2}$ Yusheng Qiu, ${ }^{1}$ and Peng $\mathrm{Xu}^{2}$ \\ ${ }^{1}$ Department of Orthopaedics, The First Affiliated Hospital, Xian Jiaotong University Health Science Center, Xian 710061, China \\ ${ }^{2}$ Department of Joint Surgery, Xian Hong Hui Hospital, Xian Jiaotong University Health Science Center, Xian 710054, China
}

Correspondence should be addressed to Yusheng Qiu; yusheng.qiu@mail.xjtu.edu.cn and Peng Xu; sousou369@163.com

Received 26 January 2016; Revised 29 May 2016; Accepted 31 May 2016

Academic Editor: Jae Youl Cho

Copyright (c) 2016 Yongsong Cai et al. This is an open access article distributed under the Creative Commons Attribution License, which permits unrestricted use, distribution, and reproduction in any medium, provided the original work is properly cited.

Juvenile idiopathic arthritis (JIA) is the most common rheumatic disease in children; some clinical trials have reported the effects of total glucosides of peony (TGP) in the treatment of JIA. However, no systematic review has yet been conducted. In this study, we assessed the efficacy and safety in patients with JIA enrolled in randomized controlled trials (RCTs) of TGP. We extracted data for studies searched from 8 electronic databases that were searched and also evaluated the methodological quality of the included studies. We assessed the following outcome measures: overall response rate, pain, tender joint count (TJC), swollen joint count (SJC), duration of morning stiffness (DMS), grip strength (GS), rheumatoid factor (RF), erythrocyte sedimentation rate (ESR), Creactive protein (CRP), and adverse effects (AEs) in short term (4-8 weeks), intermediate term (9-26 weeks), and long term (>26 weeks). The final analysis showed that TGP acted as a unique nonbiologic disease-modifying antirheumatic drug (nonbiologic DMARD), and its therapeutic effects were safe and efficacious for the treatment of JIA with few AEs. However, more high-quality RCTs are needed to confirm these therapeutic effects.

\section{Introduction}

Juvenile idiopathic arthritis (JIA), defined as unexplained joint swelling present in children under 16 years of age that persists for at least 6 weeks, is the most common rheumatic disease in children [1]. The worldwide prevalence of JIA is 7$400 / 100000$ [2]. Immune dysfunction is considered to be the key pathogenesis of the condition, but the etiology remains unclear [3]. The goal of medical management in JIA includes maximizing the protection of children's daily functional activities, relief of pain, prevention or reduction of organ damage, and minimizing drug toxicity [4]. The treatment options for JIA include nonsteroidal anti-inflammatory drugs (NSAIDs), corticosteroids, nonbiologic disease-modifying antirheumatic drugs (nonbiologic DMARDs), and biologic disease-modifying antirheumatic drugs (biologic DMARDs) (including TNF- $\alpha$ inhibitors, IL-1 inhibitors, IL- 6 inhibitors, and other biologic compounds). The successful development of biologic DMARDs has provided a more efficient method for treating JIA [5].

Paeonia lactiflora Pallas, also named Chinese Peony, is a Chinese traditional medicine. In China, Korea, and Japan, the decoction of its dried root without the bark has been used in the treatment of rheumatoid arthritis (RA) for centuries and was approved to enter the market as a nonbiologic DMARD by the State Food and Drug Administration of China in 1998. A water or ethanol extract of the root, also known as total glucosides of peony (TGP), contains more than 15 components, of which paeoniflorin is the major active ingredient [6]. In recent years, some studies have demonstrated anti-inflammatory [7-9], immunomodulatory [1012], hepatoprotective [13-18], and analgesic effects of TGP $[19,20]$ both in vitro and in vivo. The potential mechanisms of these effects include inhibition of the production of inflammatory mediators $[13,19,21]$, suppression of overactivated immune-responses, balancing the function of helper $\mathrm{T}$ cells 
(Th) and suppressor T cells (Ts), and inhibition of oxidative stress and $\mathrm{Ca}^{2+}$ overload. Some clinical trials have shown that TGP can markedly improve the quality of patients' lives and relieve the symptoms of JIA with lower incidences of side effects. However, no systematic review has yet been conducted.

The objective of this systematic review and meta-analysis is to pool the data from the included studies to examine the efficacy and safety of TGP compared with a control treatment or placebo in the treatment of JIA.

\section{Materials and Methods}

To ensure the accuracy of our systemic review and metaanalysis, we conducted our review in compliance with the Preferred Reporting Items for Systemic Reviews and MetaAnalyses (PRISMA) statement [22] as much as possible.

2.1. Eligibility Criteria. Randomized controlled trials (RCTs) that compared the effects of TGP against a control treatment (placebo, NSAIDs, DMARDs, or glucocorticoids) in people with JIA were included in this review, regardless of dates, language, blinding, or publication status. Patients must have received TGP alone or with other active drugs for a minimum of 4 weeks. JIA was diagnosed according to the 2000 International League of Associations for Rheumatology (ILAR) classification criteria [23]. TGP was defined as a water or ethanol extract of Radix Paeoniae Alba, which in this review included TGP capsules and TGP tablets. We excluded trials that used other herbal extracts, and studies without outcomes data were also eliminated.

2.2. Information Sources and Search. We searched the following electronic databases from their inception to September 30th 2015: PubMed, Cochrane Central Register of Controlled Trials, Cochrane Database of Systematic Reviews, ISI Web of knowledge, Chinese Biomedical Database (CBM), Chinese National Knowledge Infrastructure (CNKI), Wan Fang Database, and Chinese Science and Technique Journals Database (VIP). For the English databases, the search terms used were "Juvenile Idiopathic Arthritis" or "juvenile rheumatoid arthritis" or "JIA" or "JRA" or "Juvenile chronic arthritis" and "total glucosides of peony" or "total glucosides of Paeonia" or "TGP". For the Chinese databases, the key search terms were as follows: "you nian te fa xing guan jie yan" or "er tong te fa xing guan jie yan" (the Chinese name of JIA) and "bai shao zong gan" (which means total glucosides of peony) or "Pa fu lin" (a type of total glucosides of peony tablet). All search strategies were restricted to human clinical trials. To obtain any other additional articles, we manually searched all the references of the relevant studies identified. We also contacted the authors for the details of unpublished and ongoing studies.

2.3. Study Selection. Two reviewers (Yongsong Cai and Qiling Yuan) first applied the eligibility criteria independently to screen the titles and abstracts of all the records and then sought the full text of studies meeting the inclusion criteria or that were ambiguous. The kappa value was used to measure the agreement between the two reviewers. Any inconsistencies were resolved by consensus or in consultation with a third reviewer (Peng $\mathrm{Xu}$ ).

2.4. Data Collection Process. Two reviewers (Yongsong Cai and Qiling Yuan) independently extracted the data and entered it into a standard spreadsheet. The third reviewer (Jialin Zhu) verified the data accuracy. Any differences were resolved by consensus, and the authors were contacted to supplement any data lacking details.

2.5. Data Items. Information was extracted using structured data extraction tables including the following items: (1) study design; (2) patients' general data (including age, sex, diagnostic methods, subtype, and severity of JIA); (3) inclusion and exclusion criteria; (4) intervention and comparator (including dose of TGP, duration of follow-up, and type of comparator (placebo or other medicine); the duration of follow-up was defined as one of three terms: short-term (4-8 weeks), intermediate term (9-26 weeks), or long-term ( $>26$ weeks), and the latest time point would be included if there were several time points in one term); (5) outcomes including changes of effectiveness (e.g., overall response rate, pain, tender joint count (TJC), swollen joint count (SJC), duration of morning stiffness (DMS), grip strength (GS), erythrocyte sedimentation rate (ESR), C-reactive protein (CRP), and adverse effects (AEs)). Missing essential information was sought by contacting the authors.

2.6. Quality Assessment for Individual Studies. Two authors (Yongsong Cai and Qiling Yuan) independently assessed the risk of bias in the individual studies with the Cochrane Handbook for Systematic Reviews of Interventions version 5.1.0 [35] according to the following aspects: random sequence generation, blinding of participants and personnel, blinding of outcome assessment, incomplete outcome data, selective reporting, and other sources of bias. Each of the criteria was judged using three categories: low (low risk of bias), high (high risk of bias), and unclear (lack of information or uncertainty about the potential for bias). Studies with more categories judged as "low" were identified as superior. Agreement between the two authors was assessed using the kappa value. Any inconsistencies were resolved by consensus and discussion.

2.7. Data Synthesis and Analysis. Data analyses were carried out with Stata/SE version 11 (STATA Corp., College Station, TX). We pooled the data with respect to the duration of follow-up (short term, intermediate term, and long term). For dichotomous outcomes, the odds ratios (ORs) and 95\% confidence intervals (CIs) were used with the intention-totreat (ITT) principles. For continuous data, the standard mean differences (SMDs) or the mean differences (MDs) and $95 \%$ CIs were calculated. SMDs were used when the same outcomes were assessed in a variety of ways, and if the outcomes were assessed on the same scale in all trials, the MDs were adopted. We used the $I^{2}$ statistic to quantify 


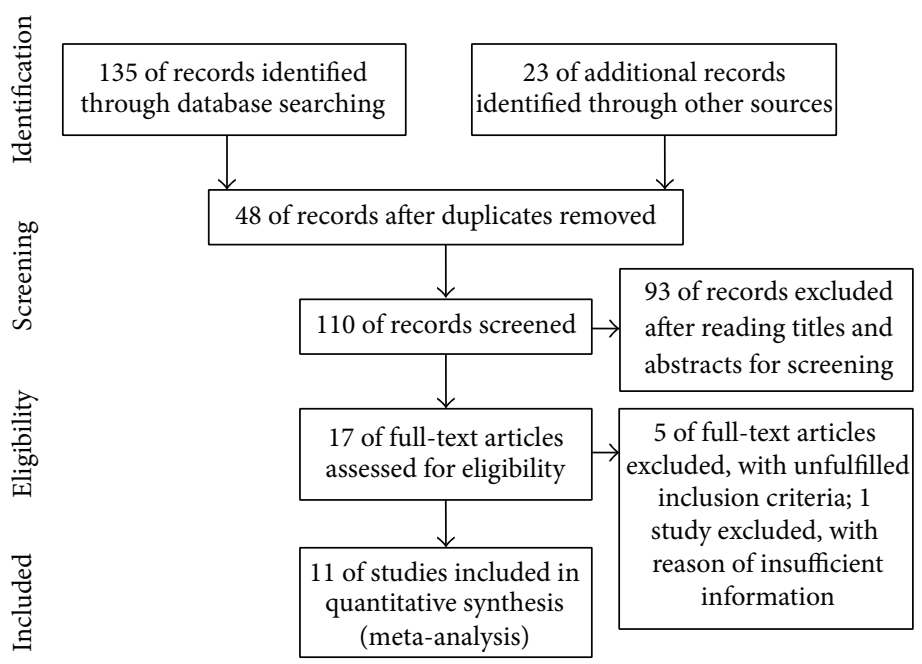

FIGURE 1: Flow diagram.

inconsistency across studies. A value of $I^{2}=0 \%$ represents no observed heterogeneity, and larger values indicate increasing heterogeneity. When $I^{2} \leq 25 \%$, we reported the pooled results using a fixed-effect model; otherwise, a randomeffects model was employed. If $I^{2}>50 \%$, we further explored the possible factors of variation. Egger's test was used to assess potential publication bias. A value of $p<0.05$ signifies the existence of potential publication bias. A sensitivity analysis was performed to assess the robustness of the results for outcomes if applicable. A $p$ value of less than 0.05 was considered significant for all analyses.

\section{Result}

3.1. Study Selection. Our initial search identified 158 studies, of which 48 duplicate studies were removed. After reading the titles and abstracts, 93 articles were excluded. Next, we read the full text of the remaining 17 articles, and 5 of them were excluded because they did not meet the inclusion criteria. One study had insufficient information (i.e., did not report any data about outcomes); the author was contacted for additional data but did not respond. Finally, 11 references [24-34] with a total of 590 participants were included (TGP: 308, control: 282) (Figure 1). Agreement on study selection between the 2 reviewers had a favorable consistency (kappa value $=0.90)$.

3.2. Study Characteristics. Table 1 listed the characteristics of the included studies that were published from 2004 to 2012. Eleven RCTs including 590 participants were conducted in China and published in Chinese. The majority of the studies were conducted as single-center trials, with only one study [27] conducted as a multicenter trial. Overall, the age of participants in the nine trials [25-29, 31-34] ranged from 1.5 to 14 years, and the average age was 8.8 years, although Shi and Ding [24] and Tong and Shui [30] did not report the average age. Males accounted for $55 \%$ of the participants in the nine [25-27, 29-34] studies, and two studies [24, 28] did not mention the gender distribution. The majority of trials showed the composition of each subtype of JIA, although five trials did not $[24,25,28,30,31]$. Two of the trials $[24,26]$ including 99 participants compared TGP alone with Methotrexate (MTX) alone. Nine studies had more than two types of treatment in the intervention or control groups as follows: TGP plus DMARDs versus DMARDs alone in 7 trials [27-29, 31-34]; TGP plus NSAIDs and glucocorticoids versus a control of NSAIDs and glucocorticoids in one trial [25]; TGP plus NSAIDs and DMARDs versus a control of NSAIDs and DMARDs in one trial [30]. Different doses of TGP, which ranged from $30 \mathrm{mg} / \mathrm{kg} / \mathrm{d}$ to $60 \mathrm{mg} / \mathrm{kg} / \mathrm{d}$, were used in these studies. Moderate doses of TGP were used in most of the studies, but one study [29] did not mention the doses used. The total daily TGP intake was divided into two to three doses. Treatment duration ranged from four weeks to 12 months. Most studies used response rate, ESR, CRP, TJC, SJC, DMS, and AEs for measuring outcomes. The response rate was defined as the proportion of the number of participants who reached an efficacy average index 30 (EAI30) in all studies except Zang [26] (EAI50) and Shi and Ding [24] (EAI20). The EAI30 was defined as an average minimum of $30 \%$ improvement from baseline in all the included outcomes. Only one study [34] used the ACR70 to evaluate the effect of TGP.

3.3. Risk of Bias within Studies. Table 2 shows the risk of bias across all studies. All the included clinical trials were associated with a high risk of bias. All the 11 included trials reported random sequence generation, but only one trial [33] provided the information regarding how randomization was performed. One trial [27] included the use of allocation concealment, and the processes used in the remaining studies were unclear. Blinding was unclear in all of the included studies. Agreement between the two authors for each aspect 


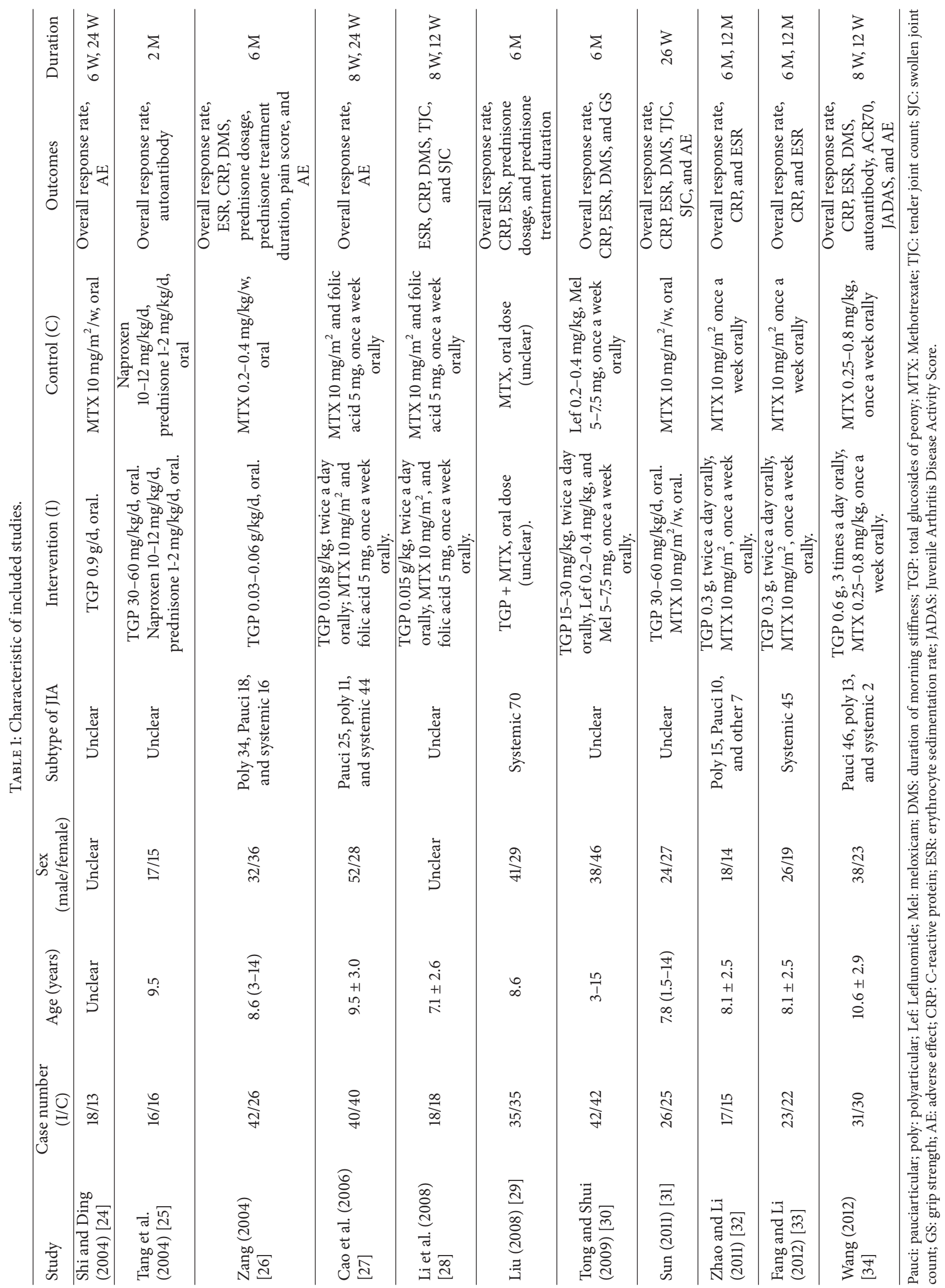


TABLE 2: Methodological quality of included trials.

\begin{tabular}{|c|c|c|c|c|c|c|c|}
\hline Author & $\begin{array}{l}\text { Random } \\
\text { sequence } \\
\text { generation }\end{array}$ & $\begin{array}{l}\text { Allocation } \\
\text { concealment }\end{array}$ & $\begin{array}{l}\text { Blinding of } \\
\text { participants and } \\
\text { personnel }\end{array}$ & $\begin{array}{l}\text { Blinding of } \\
\text { outcome } \\
\text { assessment }\end{array}$ & $\begin{array}{l}\text { Incomplete } \\
\text { outcome data }\end{array}$ & $\begin{array}{l}\text { Selective } \\
\text { reporting }\end{array}$ & $\begin{array}{c}\text { Other } \\
\text { sources of } \\
\text { bias }\end{array}$ \\
\hline $\begin{array}{l}\text { Shi and Ding } \\
\text { (2004) [24] }\end{array}$ & $\mathrm{U}$ & $\mathrm{U}$ & $\mathrm{H}$ & $\mathrm{U}$ & $\mathrm{H}$ & $\mathrm{H}$ & $\mathrm{H}$ \\
\hline $\begin{array}{l}\text { Tang et al. (2004) } \\
{[25]}\end{array}$ & $\mathrm{U}$ & $\mathrm{U}$ & $\mathrm{H}$ & $\mathrm{U}$ & $\mathrm{U}$ & $\mathrm{L}$ & $\mathrm{U}$ \\
\hline Zang (2004) [26] & $\mathrm{U}$ & $\mathrm{U}$ & $\mathrm{U}$ & $\mathrm{U}$ & $\mathrm{L}$ & $\mathrm{L}$ & $\mathrm{U}$ \\
\hline $\begin{array}{l}\text { Cao et al. (2006) } \\
\text { [27] }\end{array}$ & $\mathrm{U}$ & $\mathrm{L}$ & $\mathrm{U}$ & $\mathrm{U}$ & $\mathrm{U}$ & $\mathrm{L}$ & $\mathrm{U}$ \\
\hline Li et al. (2008) [28] & $\mathrm{U}$ & $\mathrm{U}$ & $\mathrm{U}$ & $\mathrm{U}$ & $\mathrm{H}$ & $\mathrm{H}$ & U \\
\hline Liu (2008) [29] & $\mathrm{U}$ & $\mathrm{U}$ & $\mathrm{H}$ & $\mathrm{U}$ & $\mathrm{H}$ & $\mathrm{U}$ & $\mathrm{U}$ \\
\hline $\begin{array}{l}\text { Tong and Shui } \\
\text { (2009) [30] }\end{array}$ & $\mathrm{U}$ & $\mathrm{U}$ & $\mathrm{H}$ & $\mathrm{U}$ & $\mathrm{H}$ & $\mathrm{U}$ & $\mathrm{U}$ \\
\hline Sun (2011) [31] & $\mathrm{U}$ & $\mathrm{U}$ & $\mathrm{U}$ & $\mathrm{U}$ & $\mathrm{L}$ & $\mathrm{L}$ & $\mathrm{U}$ \\
\hline $\begin{array}{l}\text { Zhao and Li (2011) } \\
\text { [32] }\end{array}$ & $\mathrm{U}$ & $\mathrm{U}$ & $\mathrm{H}$ & $\mathrm{U}$ & $\mathrm{U}$ & $\mathrm{H}$ & $\mathrm{U}$ \\
\hline $\begin{array}{l}\text { Fang and Li (2012) } \\
\text { [33] }\end{array}$ & $\mathrm{L}$ & $\mathrm{U}$ & $\mathrm{H}$ & $\mathrm{U}$ & $\mathrm{U}$ & $\mathrm{H}$ & $\mathrm{U}$ \\
\hline Wang (2012) [34] & U & $\mathrm{U}$ & $\mathrm{H}$ & $\mathrm{U}$ & $\mathrm{L}$ & $\mathrm{L}$ & $\mathrm{U}$ \\
\hline
\end{tabular}

U: unclear; H: high risk; L: low risk.

ranged from $72 \%$ to $100 \%$, and the overall agreement was $90 \%$.

3.4. Adverse Events. Five trials [24, 26, 27, 31, 34] including 157 intervention patients and 134 controls reported adverse events. The pooled OR (95\% CI) in the TGP plus MTX versus MTX alone subgroup, the TGP alone versus MTX alone subgroup, and the overall group was 0.63 (0.30 to 1.33 ), 0.14 (0.03 to 0.69 ), and 0.38 ( 0.17 to 0.82 ), respectively, which indicated that the intervention group had a lower incidence of AEs in the TGP alone versus the MTX alone subgroup and the overall group (Figure 2 and Table 3). The main AEs in intervention were gastrointestinal events which included nausea, abdominal distention, anorexia, and diarrhea. In the control group, the main AEs included liver function abnormalities, leucopenia, rash, stomatitis, and anorexia. The majority of these events were mild to moderate in intensity and did not require treatment.

\section{Intervention Efficacy}

4.1. Cointervention versus Control. Nine trials compared a cointervention of TGP and DMARDs or NSAIDs or glucocorticoids with a control as follows: TGP plus DMARDs versus DMARDs alone in 7 trials [27-29, 31-34]; TGP plus NSAIDs and glucocorticoids versus a control of NSAIDs and glucocorticoids in one trial [25]; TGP plus NSAIDs and DMARDs versus a control of NSAIDs and DMARDs in one trial [30].

4.1.1. The Overall Response Rate. Seven studies $(n=423)$ compared a cointervention and a control group [27, 29-34]. The overall response rates were assessed at each treatment term, which included short term (4-8 weeks), intermediate term (9-26 weeks), and long term ( $>26$ weeks), and the overall response rate at each term was as follows: $\mathrm{OR}=1.55$, 95\% CI: 0.68 to 3.51 ; OR $=2.47,95 \%$ CI: 1.22 to 5.0 ; and OR $=2.11,95 \%$ CI: 0.56 to 7.93 , respectively, with no significant heterogeneity $\left(I^{2}=0 \%\right)$, which indicated that cointervention was favored in the overall response rate in the intermediate term (Figure 3). Sensitivity analysis was performed by using jackknife analysis in the intermediate term and, in most cases, removal of a study did not significantly change the result (OR ranged from 2.27 to $2.86, p<0.03$ ), except in the study by Tong (OR $=2.04 ; 95 \% \mathrm{CI}$ : 0.93 to 4.47 ; $p=0.074$ ) (Table 3 and Supplementary Materials available online at http://dx.doi.org/10.1155/2016/8292486). Egger's test indicated no significant publication bias (coefficient $=0.59$; $\mathrm{SE}=0.90 ; t=0.65 ; p=0.545)$ in the intermediate term (Supplementary Materials).

4.1.2. ESR. Cointervention showed very large treatment effects for ESR compared to DMARDs alone [28, 29, 31-34] or DMARDs plus NSAIDs [30] $(n=379)$. The pooled MD for ESR was $-4.21 \mathrm{~mm} / \mathrm{h}(95 \% \mathrm{CI}$ : -9.31 to $0.89 \mathrm{~mm} / \mathrm{h})$ in the short term, $-3.36 \mathrm{~mm} / \mathrm{h}(95 \% \mathrm{CI}:-5.18$ to $-1.54 \mathrm{~mm} / \mathrm{h})$ in the intermediate term, and $-3.72 \mathrm{~mm} / \mathrm{h}$ (95\% CI: -6.66 to $-0.78 \mathrm{~mm} / \mathrm{h}$ ) in the long term. That is, the ESR in the cointervention groups was $3.36 \mathrm{~mm} / \mathrm{h}$ lower than the ESR of the control groups in the intermediate term and $3.72 \mathrm{~mm} / \mathrm{h}$ lower than the control groups at the long term, but heterogeneity existed in the intermediate term $\left(I^{2}=58.1 \%, p=0.026\right)$ and long term $\left(I^{2}=91.7 \%, p=0.001\right)$ (Figure 4$)$. The source of the heterogeneity in the intermediate term was not apparent. A sensitivity analysis was performed by pooling the 6 studies [28-30, 32-34] with cointerventions versus DMARDs in 


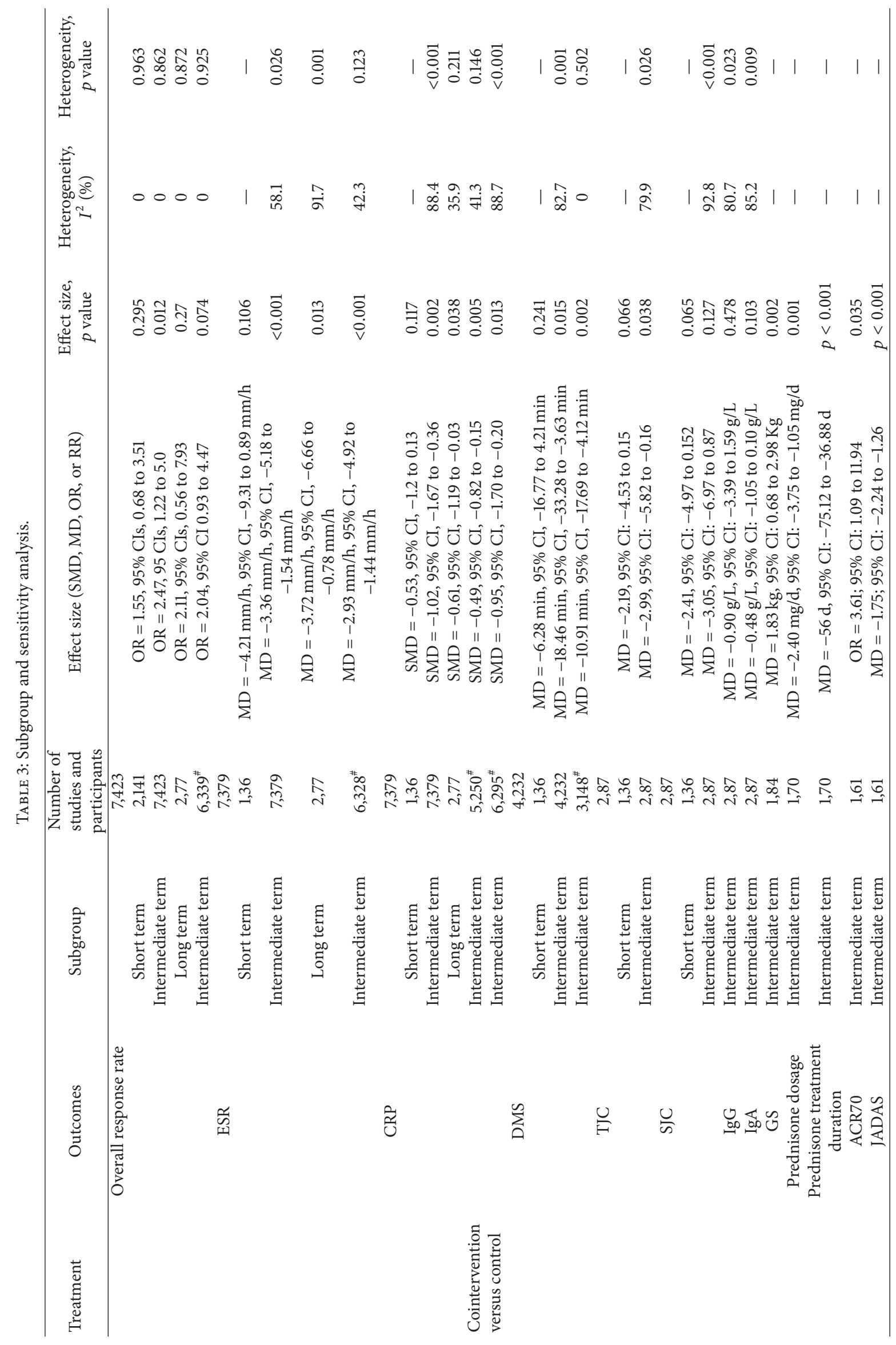




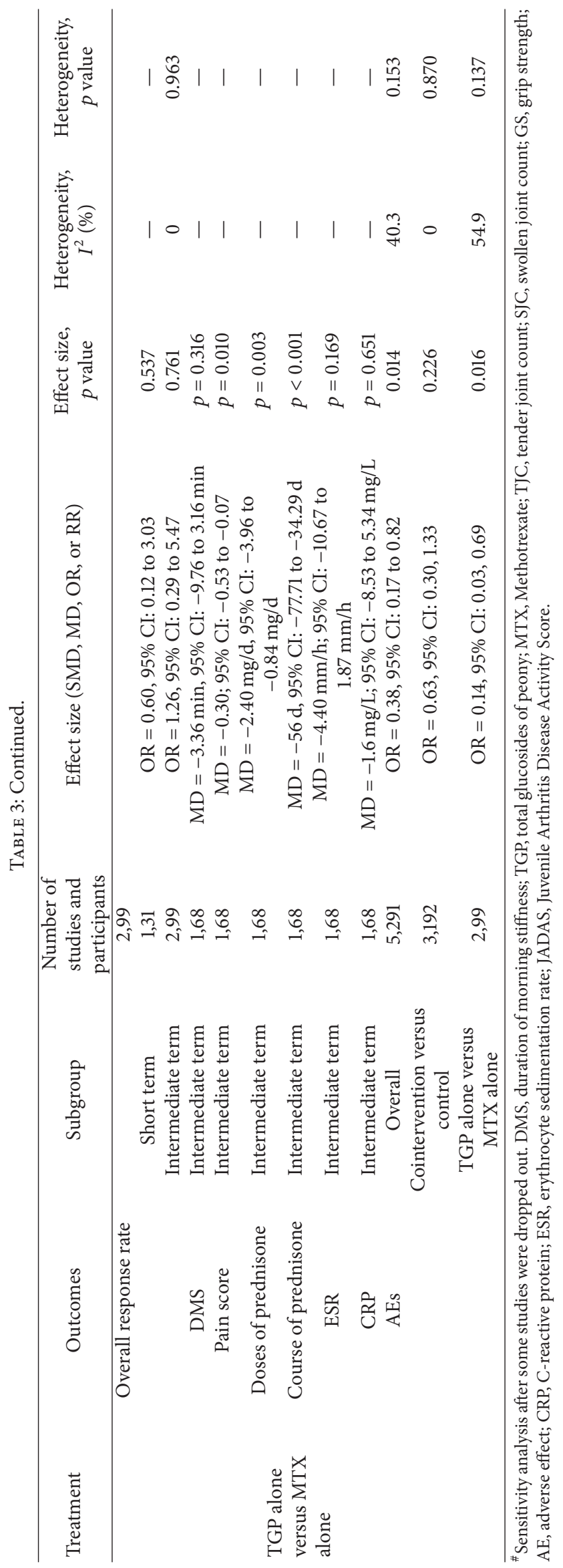




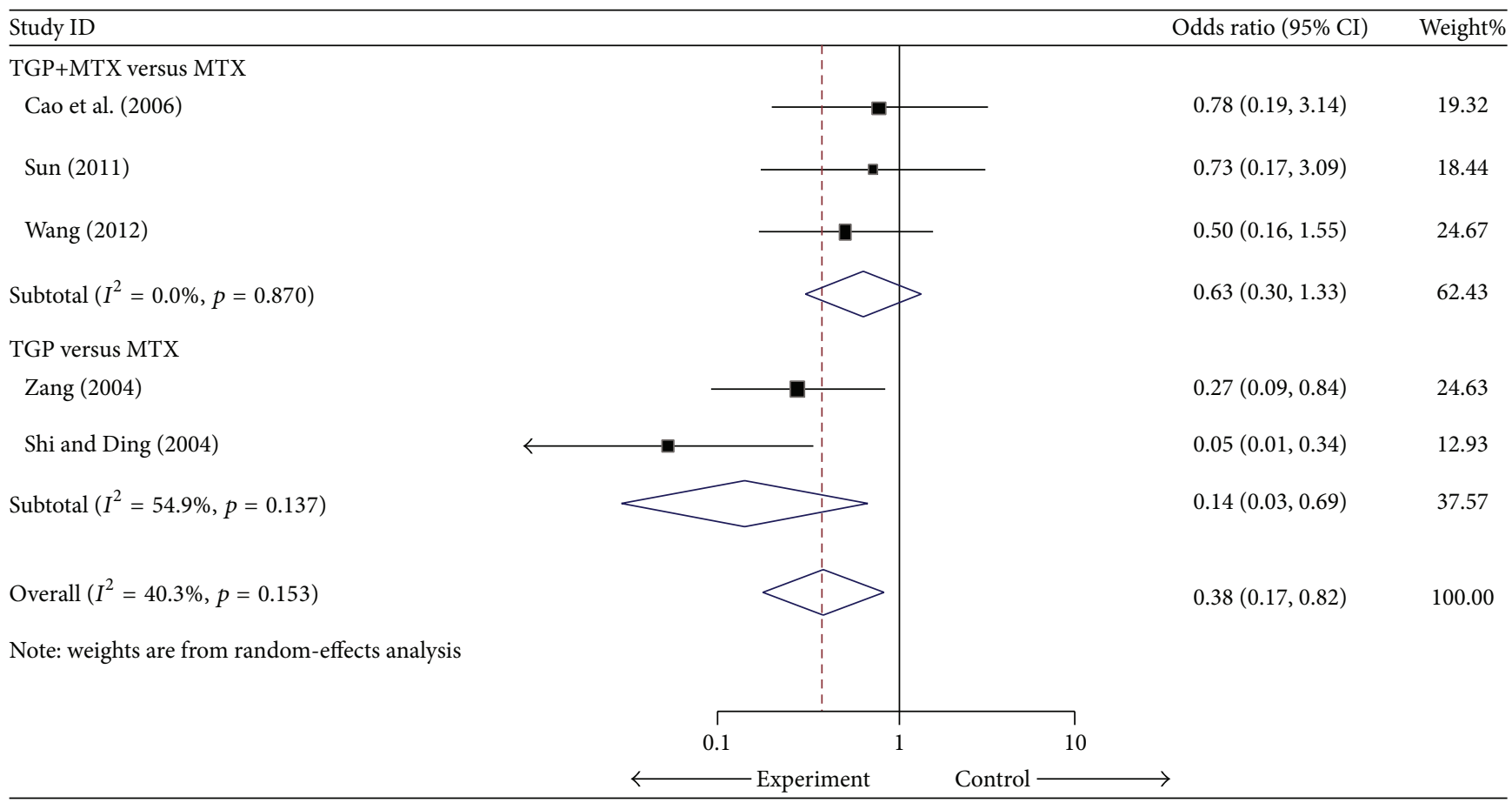

FIGURE 2: Forest plot of the random-effects model of the odds ratio (95\% CI) in the AEs for TGP versus controls.

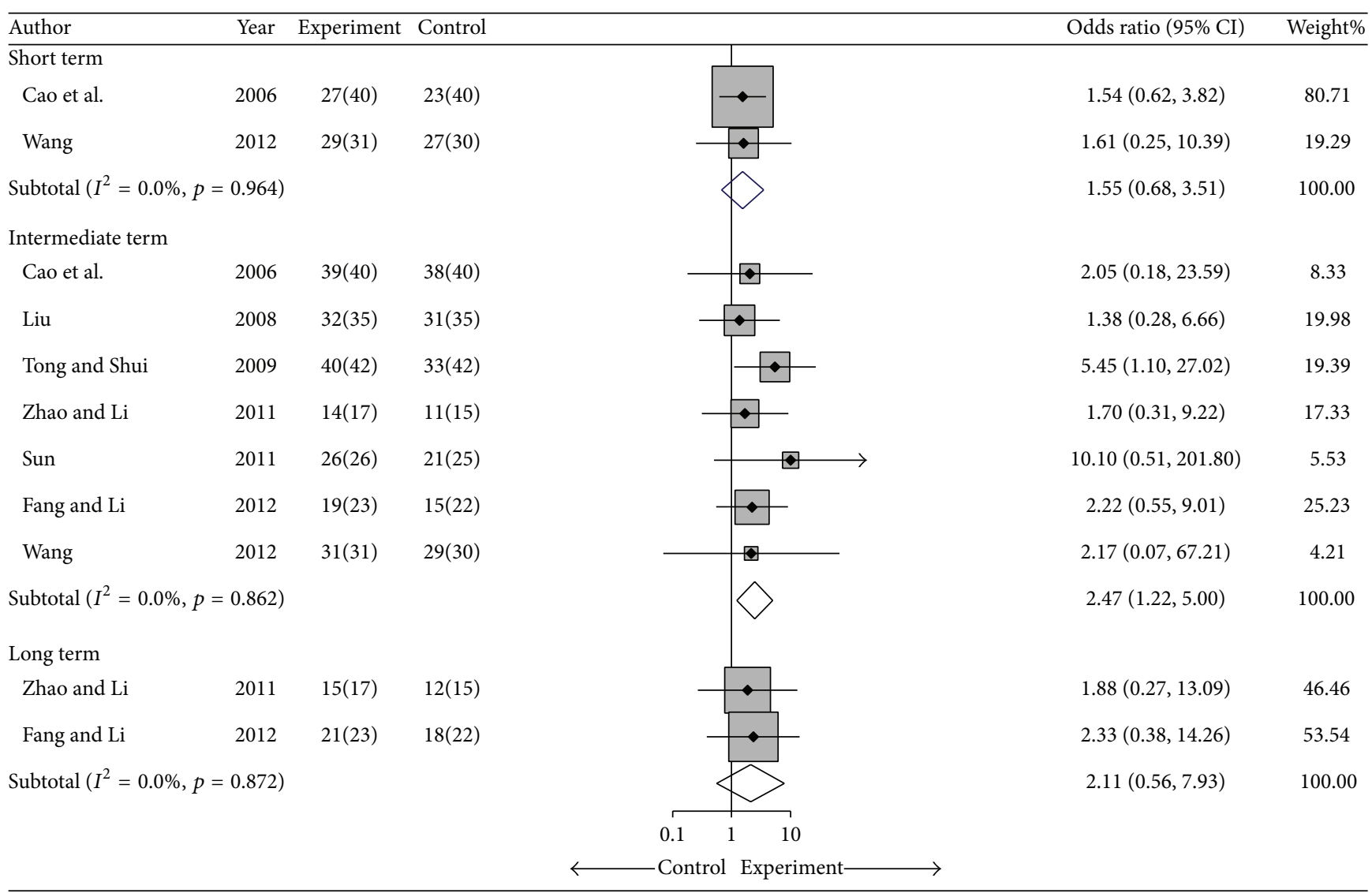

FIGURE 3: Forest plot of the fixed-effects model of the odds ratio (95\% CI) in the overall response rate for cointervention versus controls in short term, intermediate term, and long term. 


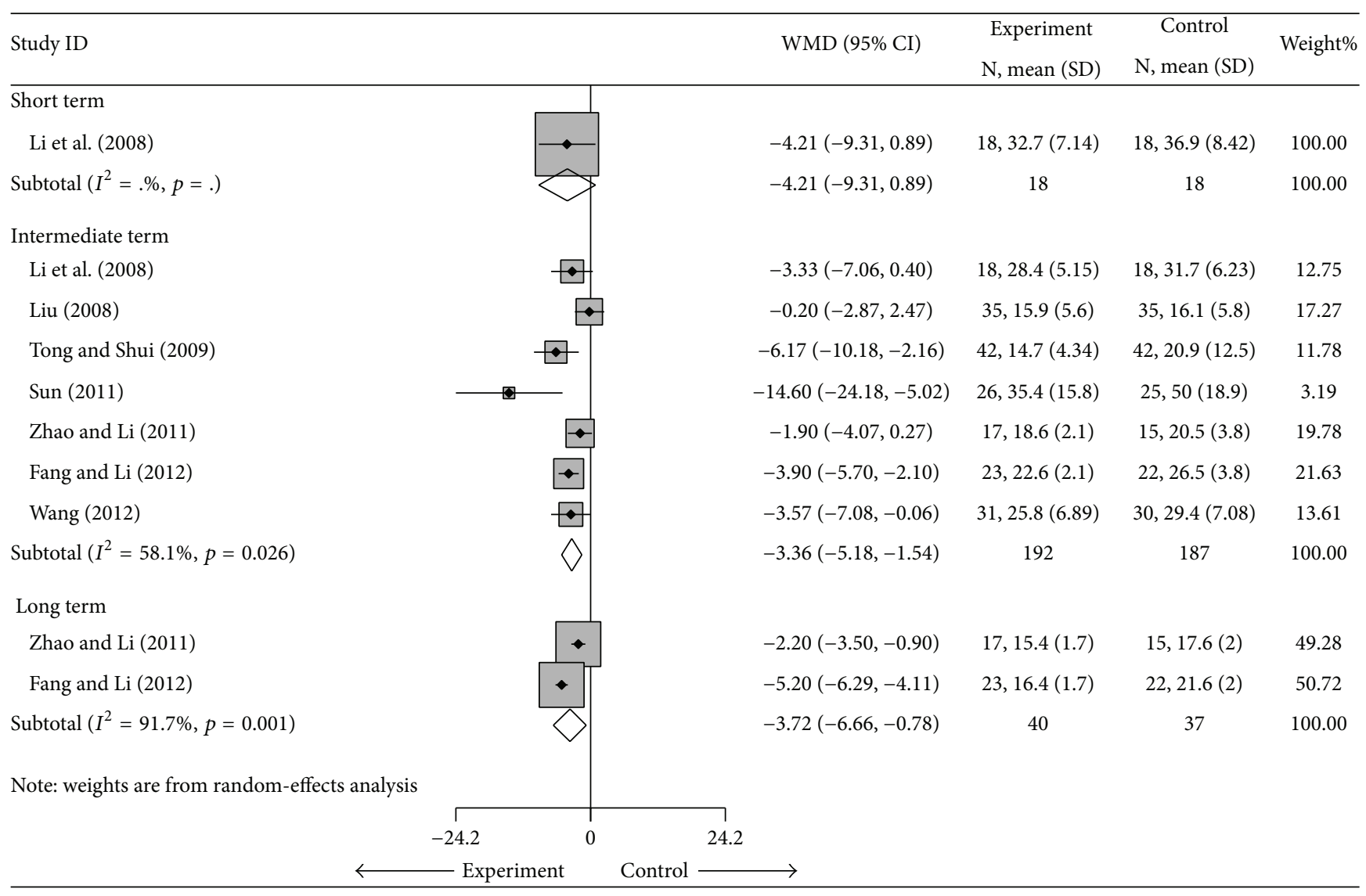

FIGURE 4: Forest plot of the random-effects model of the MD (95\% CI) in the ESR for cointervention versus controls in short term, intermediate term, and long term.

the intermediate term, and the treatment effect was still significant, with an $\mathrm{MD}$ of $-2.93 \mathrm{~mm} / \mathrm{h}(95 \% \mathrm{CI}:-4.92$ to $-1.44 \mathrm{~mm} / \mathrm{h}$ ) (Table 3 and Supplementary Materials). Egger's test indicated no significant publication bias (coefficient $=$ -2.03; SE $=1.38 ; t=-1.47 ; p=0.203$ ) in the intermediate term (Supplementary Materials).

4.1.3. CRP. Seven studies [28-34] with a total of 379 participants compared the CRP between the cointervention groups and the groups using DMARDs alone [28, 29, 31-34] or DMARDs plus NSAIDs [30]. In the random-effects model, the SMDs in the short term, intermediate term, and long term were -0.53 (95\% CI: -1.2 to 0.13 ), -1.02 (95\% CI: -1.67 to -0.36 ), and -0.61 (95\% CI: -1.19 to -0.03 ), respectively. That is, patients in the cointervention groups had a 1.02-unit significantly lower CRP in intermediate term and 0.61-unit lower CRP in long term than the patients in the control group (Figure 5). There was high heterogeneity in the intermediate term $\left(I^{2}=88.4 \%\right)$. The source of this heterogeneity was unclear, but when two studies were removed [30, 33], the SMD was -0.49 (95\% CI: -0.82 to $-0.15, I^{2}=41.3 \%$ ) (Table 3 and Supplementary Materials). In the sensitivity analysis limited to the 6 studies [28, 29, 31-34] with cointervention versus DMARDs in intermediate term, the SMD was -0.95 (95\% CI: -1.7 to -0.2 ) (Table 3 and Supplementary Materials).
No significant publication bias was found by Egger's test (coefficient $=-7.5 ; \mathrm{SE}=4.66 ; t=-1.62 ; p=0.166)$ in intermediate term (Supplementary Materials).

4.1.4. DMS. Four studies $[28,30,31,34](n=232)$ compared cointervention with DMARDs alone [28, 31, 34] or DMARDs plus NSAIDs [30] for the outcome of DMS and had a pooled $\mathrm{MD}$ of $-6.28 \mathrm{~min}$ ( $95 \% \mathrm{CI},-16.77$ to $4.21 \mathrm{~min}$ ) in short term and $-18.46 \mathrm{~min}$ ( $95 \% \mathrm{CI},-33.28$ to $-3.63 \mathrm{~min})$ in intermediate term (Figure 6). The results indicated that cointervention had a shorter DMS than the control group in intermediate term. However, the results were heterogenous $\left(I^{2}=82.7 \%\right)$. Sensitivity analysis was performed using the 3 studies [28, 31, 34] with cointervention versus DMARDs alone; the MD was $-10.91 \mathrm{~min}$ (95\% CI: -17.69 to -4.21$)$ and $I^{2}=0 \%$, which indicated that Tong [30] was the main source of this heterogeneity (Table 3 and Supplementary Materials). There was no evidence of publication bias (coefficient $=-0.07$, $\mathrm{SE}=4.7, t=-0.01, p=0.99$ ) (Supplementary Materials).

4.1.5. TJC. Two studies $[28,31]$ with a total of 87 patients compared TJC between the cointervention group and DMARDs alone. The MD was -2.19 (95\% CI: -4.53 to 0.15 ) in short term and -2.99 ( $95 \% \mathrm{CI}:-5.82$ to -0.16$)$ in intermediate term, meaning that the cointervention group had less TJC than 


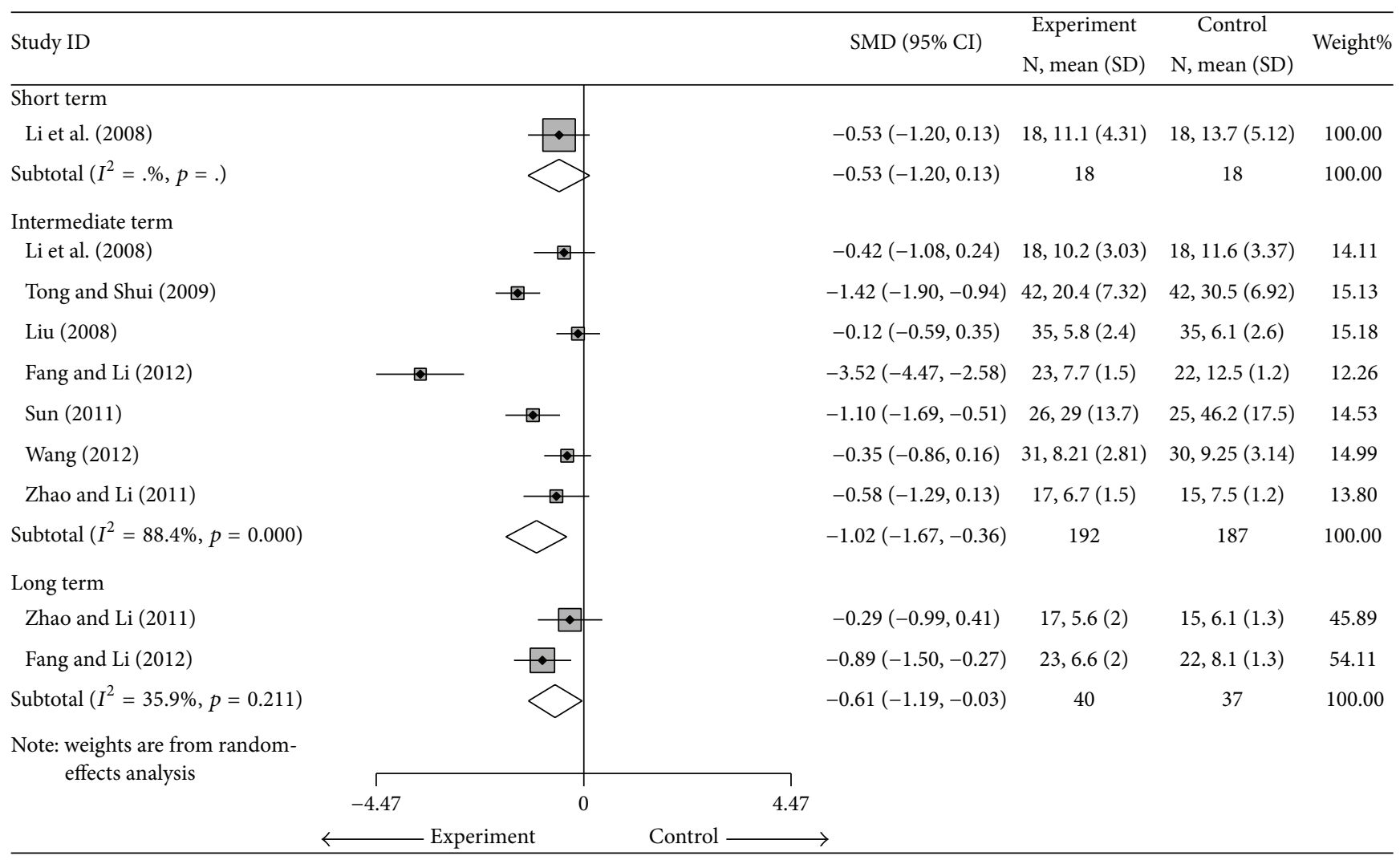

FIGURE 5: Forest plot of the random-effects model of the SMD (95\% CI) in the CRP for cointervention versus controls in short term, intermediate term, and long term.

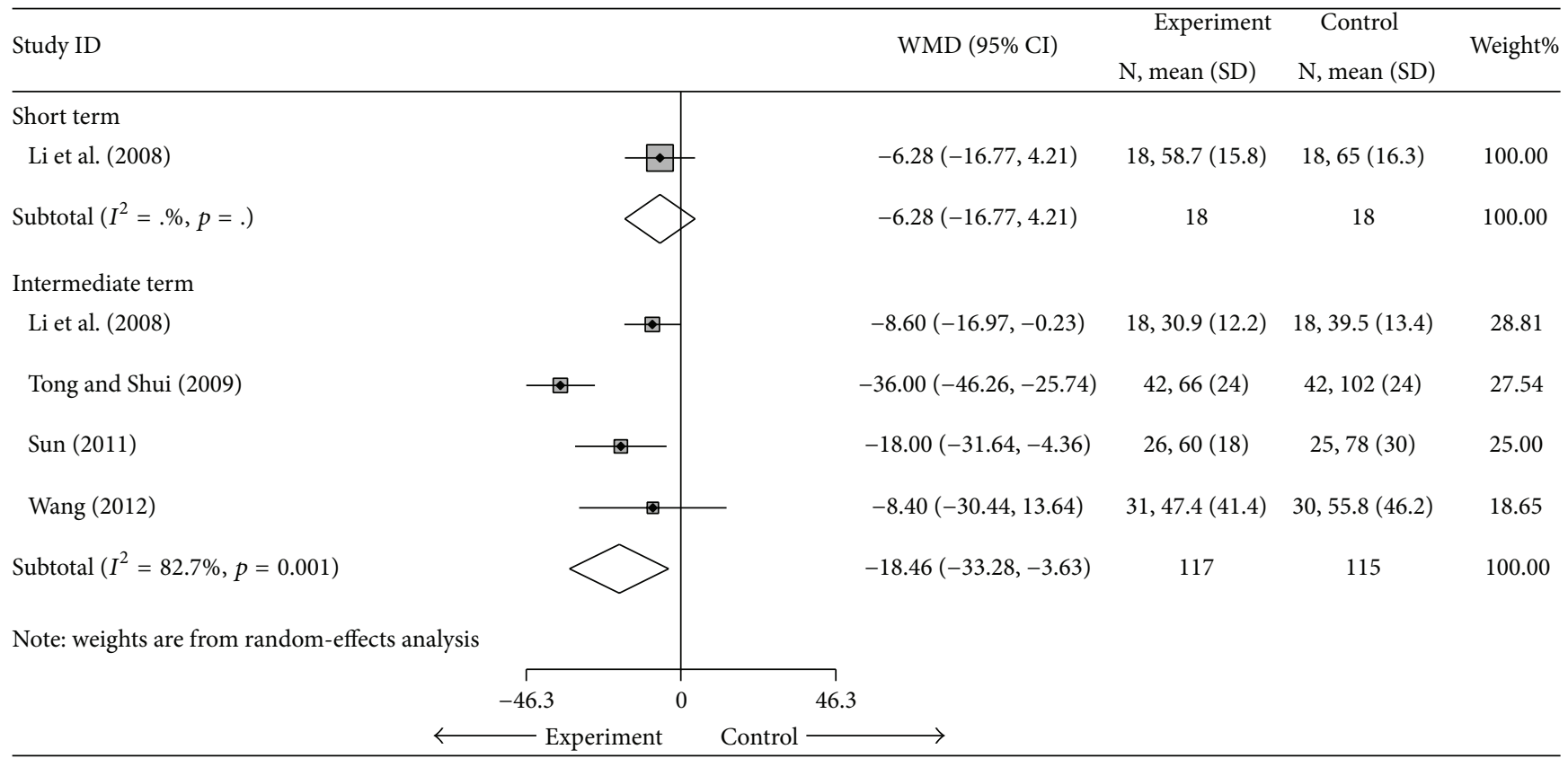

FIGURE 6: Forest plot of the random-effects model of the MD (95\% CI) in the DMS for cointervention versus controls in short term and intermediate term. 
the DMARD only group in intermediate term (Table 3 and Supplementary Materials).

4.1.6. SJC. Two studies $[28,31]$ compared SJC between the cointervention group and the group using DMARDs alone $(n=87)$. No effect was found in short term (MD $=-2.41 ; 95 \%$ CI: -4.97 to 0.152$)$ and intermediate term (MD $=-3.05 ; 95 \%$ CI: -6.97 to 0.87 ) (Table 3 and Supplementary Materials).

4.1.7. Autoantibodies. Two studies $(n=93)[25,34]$ compared IgG and IgA, and the pooled MDs were -0.90 (95\% CI: -3.39 to 1.59 ) and -0.48 (95\% CI: -1.05 to 0.10 ), respectively (Table 3 and Supplementary Materials).

4.1.8. Other. Tong and Shui [30] compared GS in intermediate term and the pooled MD was $1.83 \mathrm{~kg}$ (95\% CI: 0.68 to 2.98; $p=0.002$ ); Liu [29] compared the prednisone dosage and prednisone treatment duration and the pooled MD was $-2.40 \mathrm{mg} / \mathrm{d}$ (95\% CI: -3.75 to $-1.05 ; p=0.001)$ and $-56 \mathrm{~d}$ (95\% CI: -75.12 to $-36.88 ; p<0.001$ ), respectively. Only one study [34] described the response rate of ACR70 (OR = 3.61; 95\% CI: 1.09 to 11.94; $p=0.04)$ and Juvenile Arthritis Disease Activity Score (JADAS) (MD $=-1.75$; 95\% CI: -2.24 to -1.26 ; $p<0.001$ ) (Table 3 and Supplementary Materials).

\subsection{TGP Compared with DMARDs Alone}

4.2.1. The Overall Response Rate. Two studies $[24,26]$ with a total of 99 patients were pooled to compare TGP alone with MTX alone. The OR was 0.60 (95\% CI: 0.12 to 3.03) in short term and 1.26 (95\% CI: 0.29 to 5.47) in intermediate term with no significant heterogeneity $\left(I^{2}=0 \%\right)$. That is, patients receiving TGP alone had a better response rate than patients who received MTX alone, but this finding did not reach statistical significance ( $p=0.761$ in intermediate term) (Table 3 and Supplementary Materials).

Only one study [26] described the result of continuous data in TGP versus MTX alone group, and the duration of treatment was 6 months. A significant difference was identified between TGP and the MTX alone group in terms of the pain score ( $p=0.010 ; \mathrm{MD}=-0.30 ; 95 \% \mathrm{CI}:-0.53$ to $-0.07)$, dose of prednisone $(p=0.003 ; \mathrm{MD}=-2.40 \mathrm{mg} / \mathrm{d}$; 95\% CI: -3.96 to -0.84$)$, and course of prednisone ( $p<$ $0.001 ; \mathrm{MD}=-56 \mathrm{~d} ; 95 \% \mathrm{CI}:-77.71$ to -34.29$)$, signifying that the TGP group could shorten the course of prednisone, alleviate pain, and decrease the dose of prednisone. However, no effects were found in DMS ( $p=0.316 ; \mathrm{MD}=-3.36 \mathrm{~min}$; $95 \%$ CI: -9.76 to 3.16 ), ESR ( $p=0.169 ; \mathrm{MD}=-4.40 \mathrm{~mm} / \mathrm{h}$; $95 \%$ CI: -10.67 to 1.87$)$, or CRP $(p=0.651 ; \mathrm{MD}=-1.6 \mathrm{mg} / \mathrm{L}$; $95 \%$ CI: -8.53 to 5.34 ) (Table 3 ).

\section{Discussion}

To the best of our knowledge, this is the first systematic review and meta-analysis evaluating the efficacy and safety of TGP for the treatment of JIA. We pooled the results from 11 studies including 590 participants. To minimize the heterogeneity, three subgroups were set according to the treatment duration. The relevant clinical outcomes were studied, including ESR, CRP, DMS, SJC, TJC, autoantibodies, and treatment response rates, as well as other relevant clinical outcomes (i.e., GS, ACR70, pain score, and DAS). Overall, the results of this meta-analysis suggested favorable effects of TGP plus DMARDs or NSAIDs in patients with JIA in intermediate term $(9-26 \mathrm{~W})$, and the overall incidence of AEs was lower in intervention group. However, statistical significance was not attained in most of the results in short term and long term, especially in the TGP alone versus DMARD alone groups, which might be explained by the lack of sufficient studies included. In addition, the overall methodological quality was low. Therefore, caution should be exercised in interpreting these positive results.

In this meta-analysis, TGP combined with DMARDs or NSAIDs showed better effects on the ESR, CRP, DMS, and treatment response rates. Another highlight of our study was the finding that the intervention groups had a lower incidence of AEs ( $R R=0.58,95 \%$ CI: 0.38 to 0.91 ), particularly the incidence of hepatotoxicity (2/308 versus $6 / 282, \mathrm{RR}=0.31,95 \% \mathrm{CI}: 0.06$ to 1.52 ). Some findings might explain the potential therapeutic effect of TGP in the treatment of JIA. Preclinical studies have shown that TGP displays immune-regulatory, anti-inflammatory, and hepatoprotective activities. In experimental arthritis rats, intragastric administration of TGP $(25-100 \mathrm{mg} / \mathrm{kg} / \mathrm{d})$ for $7-$ 14 days was demonstrated to notably diminish the severity of hind paw swelling and the scores of polyarthritis in a dose-dependent manner [7-10, 12, 36, 37]. The synovial infiltration of lymphocytes $[8,9,12]$, hypertrophy of synovial membranes, and the formation of new blood vessels and pannus were also significantly inhibited by TGP $[8,9]$. In vitro, paeoniflorin could induce apoptosis in murine $\mathrm{T}$ lymphocytes and Jurkat human $\mathrm{T}$ cell leukemia cells [11]. The most exciting effect of TGP was its hepatoprotective activity. Paeoniflorin has been shown to protect mice against Schistosoma japonicum egg-induced hepatic fibrosis by interfering with the IL-13 signaling molecule and decreasing the level of IL-13 [38, 39]. Other studies have also shown that paeoniflorin could protect mice against Bacillus CalmetteGuerin or lipopolysaccharide-induced liver injury [14, 40], which might be associated with the effects of inhibition of IL$1 \beta$ and TNF- $\alpha$ release and the promotion of IL-10 production [21]. Recent research has also suggested that TGP could reduce the hepatotoxicity caused by combination treatment with Methotrexate and Leflunomide for active rheumatoid arthritis [18]. All the data supported the potential therapeutic effect of TGP in the treatment of JIA.

Three meta-analyses have been published on the efficacy and safety of TGP for the treatment of RA [41-43], but all of them have been published in Chinese. Among the three meta-analyses, two of them $[41,42]$ drew positive conclusions with the OR of TGP versus the control group for the overall response rates, which were 1.13 (95\% CI: 1.04 to 1.24 ) and 2.65 (95\% CI: 1.51 to 4.65), respectively. One trial [43] drew negative conclusions with the OR for the overall response rate $(\mathrm{RR}=3.41,95 \% \mathrm{CI}: 0.77$ to 15.10$)$. The incidence of AEs in all the trials ranged from 0.41 to 0.66 , and the incidence of hepatotoxicity ranged from 0.19 to 0.41 . Most of the included 
studies indicated that TGP demonstrates beneficial effects on the symptoms of RA with a lower incidence of AEs, especially the incidence of hepatotoxicity. However, all of them also found that the incidence of diarrhea was higher in patients treated with TGP than in patients who were not treated with TGP. Our study resulted in similar conclusions. Considering that the treated subjects are children, the incidence of diarrhea should be noted when using TGP for the treatment of JIA.

The results of our meta-analysis should be treated with caution for several reasons. First, all the included trials were conducted in China, which was a potential selection bias, and might influence the application of TGP for patients in other countries. Second, the selected trials did not report ethical approval, which may weaken the validity of the results. Third, the methodological quality of the included studies was poor; while all of the selected studies were described as randomized controlled trials, only one trial [33] provided the information regarding how randomization was performed. Only one trial mentioned allocation concealment [27], and the blinding was unclear in all of the included studies. Fourth, in our study, we pooled the results without regard for the subtype of JIA, because treatment according to subtypes was not used in most of the included trials, which may affect the applicability for particular subtypes. Fifth, small sample sizes, insufficient numbers of included trials in each subgroup, and extensive heterogeneity in some results weaken the overall results of the review. Sixth, except for CRP, there was no other inflammatory factor, such as TNF- $\alpha$ or PGE2, which may weaken the persuasive. Lastly, the outcome measures used were inadequate, as only one trial used the American College of Rheumatology Pediatric (ACR Pedi) response criteria [34] and the Juvenile Arthritis Disease Activity Score (JADAS), and none of the included studies used tools for the assessment of quality of life, which is very important for evaluating the therapeutic effect, such as the Childhood Health Assessment Questionnaire (CHAQ), Child Health Questionnaire (CHQ), and Pediatric Quality of Life (PedsQL) [5]. All of these limitations may influence the application of TGP for treatment of JIA and weaken the validity of the results.

To effectively compare treatment options in different countries, future clinical RCTs should follow standardized and validated outcome measures and use accepted standards of trial design and reporting (CONSORT).

In conclusion, TGP is safe and efficacious for the treatment of JIA with few AEs. TGP, which has similar effects on nonbiologic DMARDs, is a special type of nonbiologic DMARD for the treatment of JIA.

\section{Competing Interests}

All authors declare that there are no competing interests regarding the publication of this paper.

\section{Acknowledgments}

This work was supported by the National Natural Science Foundations of China (no. 81271948 and no. 81171742).

\section{References}

[1] R. E. Petty, T. R. Southwood, P. Manners et al., "International league of associations for rheumatology classification of juvenile idiopathic arthritis: second revision, Edmonton, 2001," Journal of Rheumatology, vol. 31, no. 2, pp. 390-392, 2004.

[2] P. J. Manners and C. Bower, "Worldwide prevalence of juvenile arthritis-why does it vary so much?" Journal of Rheumatology, vol. 29, no. 7, pp. 1520-1530, 2002.

[3] L. Adorini and G. Penna, "Control of autoimmune diseases by the vitamin D endocrine system," Nature Clinical Practice Rheumatology, vol. 4, no. 8, pp. 404-412, 2008.

[4] L. Kroger, P. Vahasalo, P. Tynjala et al., "Medical treatment of juvenile idiopathic arthritis," Duodecim, vol. 128, no. 5, pp. 477486, 2012.

[5] E. A. Kessler and M. L. Becker, "Therapeutic advancements in juvenile idiopathic arthritis," Best Practice \& Research: Clinical Rheumatology, vol. 28, no. 2, pp. 293-313, 2014.

[6] W. Zhang and S.-M. Dai, "Mechanisms involved in the therapeutic effects of Paeonia lactiflora Pallas in rheumatoid arthritis," International Immunopharmacology, vol. 14, no. 1, pp. 27-31, 2012.

[7] H.-M. Xu, W. Wei, X.-Y. Jia, Y. Chang, and L. Zhang, "Effects and mechanisms of total glucosides of paeony on adjuvant arthritis in rats," Journal of Ethnopharmacology, vol. 109, no. 3, pp. 442448, 2007.

[8] Y.-Q. Zheng and W. Wei, “Total glucosides of paeony suppresses adjuvant arthritis in rats and intervenes cytokinesignaling between different types of synoviocytes," International Immunopharmacology, vol. 5, no. 10, pp. 1560-1573, 2005.

[9] L. Zhu, W. Wei, Y.-Q. Zheng, and X.-Y. Jia, "Effects and mechanisms of total glucosides of paeony on joint damage in rat collagen-induced arthritis," Inflammation Research, vol. 54, no. 5, pp. 211-220, 2005.

[10] H. Wu, W. Wei, L. Song, L. Zhang, Y. Chen, and X. Hu, "Paeoniflorin induced immune tolerance of mesenteric lymph node lymphocytes via enhancing beta 2-adrenergic receptor desensitization in rats with adjuvant arthritis," International Immunopharmacology, vol. 7, no. 5, pp. 662-673, 2007.

[11] H. Tsuboi, K. Hossain, A. A. Akhand et al., "Paeoniflorin induces apoptosis of lymphocytes through a redox-linked mechanism," Journal of Cellular Biochemistry, vol. 93, no. 1, pp. 162-172, 2004.

[12] Q.-T. Wang, L.-L. Zhang, H.-X. Wu, and W. Wei, “The expression change of $\beta$-arrestins in fibroblast-like synoviocytes from rats with collagen-induced arthritis and the effect of total glucosides of paeony," Journal of Ethnopharmacology, vol. 133, no. 2, pp. 511-516, 2011.

[13] I. D. Kim and B. J. Ha, "Paeoniflorin protects RAW 264.7 macrophages from LPS-induced cytotoxicity and genotoxicity," Toxicology in Vitro, vol. 23, no. 6, pp. 1014-1019, 2009.

[14] I. D. Kim and B. J. Ha, "The effects of paeoniflorin on LPSinduced liver inflammatory reactions," Archives of Pharmacal Research, vol. 33, no. 6, pp. 959-966, 2010.

[15] Q.-Q. Mao, X.-M. Zhong, Z.-Y. Li, and Z. Huang, "Paeoniflorin protects against NMDA-induced neurotoxicity in PC12 cells via $\mathrm{Ca}^{2+}$ antagonism," Phytotherapy Research, vol. 25, no. 5, pp. 681685, 2011.

[16] J. Su, P. Zhang, J.-J. Zhang, X.-M. Qi, Y.-G. Wu, and J.-J. Shen, "Effects of total glucosides of paeony on oxidative stress in the kidney from diabetic rats," Phytomedicine, vol. 17, no. 3-4, pp. 254-260, 2010. 
[17] Z. Chen, X.-P. Li, Z.-J. Li, L. Xu, and X.-M. Li, "Reduced hepatotoxicity by total glucosides of paeony in combination treatment with leflunomide and methotrexate for patients with active rheumatoid arthritis," International Immunopharmacology, vol. 15, no. 3, pp. 474-477, 2013.

[18] N. Xiang, X.-M. Li, M.-J. Zhang et al., "Total glucosides of paeony can reduce the hepatotoxicity caused by Methotrexate and Leflunomide combination treatment of active rheumatoid arthritis," International Immunopharmacology, vol. 28, no. 1, pp. 802-807, 2015.

[19] B. Lee, Y.-W. Shin, E.-A. Bae et al., "Antiallergic effect of the root of Paeonia lactiflora and its constituents paeoniflorin and paeonol," Archives of Pharmacal Research, vol. 31, no. 4, pp. 445450, 2008.

[20] X.-J. Zhang, H.-L. Chen, Z. Li et al., "Analgesic effect of paeoniflorin in rats with neonatal maternal separation-induced visceral hyperalgesia is mediated through adenosine $\mathrm{Al}$ receptor by inhibiting the extracellular signal-regulated protein kinase (ERK) pathway," Pharmacology Biochemistry and Behavior, vol. 94, no. 1, pp. 88-97, 2009.

[21] W. Cao, W. Zhang, J. Liu et al., "Paeoniflorin improves survival in LPS-challenged mice through the suppression of TNF$\alpha$ and IL- $1 \beta$ release and augmentation of IL-10 production," International Immunopharmacology, vol. 11, no. 2, pp. 172-178, 2011.

[22] A. Liberati, D. G. Altman, J. Tetzlaff et al., "The PRISMA statement for reporting systematic reviews and meta-analyses of studies that evaluate health care interventions: explanation and elaboration," Annals of Internal Medicine, vol. 151, no. 4, pp. W65-W94, 2009.

[23] I. Foeldvari and M. Bidde, "Validation of the proposed ILAR classification criteria for juvenile idiopathic arthritis," Journal of Rheumatology, vol. 27, no. 4, pp. 1069-1072, 2000.

[24] Y. Shi and X. Ding, "Observation on effectiveness of MTX and TGP on rheumatic arthritis with children," Anhui Medical and Pharmaceutical Journal, vol. 8, no. 6, pp. 413-414, 2004 (Chinese).

[25] X.-M. Tang, P.-J. Xu, Q. Li et al., “The evaluation of renal damage of total glucosides of paeony on juvenile idiopathic arthritis," Chinese Journal of Integrated Traditional and Western Nephrology, vol. 5, no. 11, pp. 646-647, 2004 (Chinese).

[26] Y.-S. Zang, "Observation on curative effects of total glucosides of paeony for juvenile idiopathic arthritis," Anhui Medical and Pharmaceutical Journal, vol. 9, no. 2, pp. 97-98, 2004 (Chinese).

[27] L.-F. Cao, Y. Zhao, H.-Y. Mao et al., "The clinical efficacy and safety of TGP treatment in juvenile idiopathic arthritis," Chinese Journal of Rheumatology, vol. 10, no. 2, pp. 100-103, 2006 (Chinese).

[28] Y. Li, L. Yang, and Z.-H. Liao, "The therapeutic effect of total glucosides of paeony combined methotrexate on juvenile idiopathic arthritis," Modern Journal of Integrated Chinese Traditional and Western Medicine, vol. 17, no. 21, pp. 3291-3292, 2008 (Chinese).

[29] X.-H. Liu, "The therapeutic effect of total glucosides of paeony combined methotrexate on systemic juvenile idiopathic arthritis," Jiangxi Zhong Yi Yao, vol. 39, no. 305, pp. 37-38, 2008 (Chinese).

[30] H.-Y. Tong and Y.-X. Shui, "The effect of total glucosides of paeony on juvenile idiopathic arthritis," Zhejiang Zhongyi Za Zhi, vol. 44, no. 7, p. 543, 2009 (Chinese).

[31] Y. Sun, "Comparative study on clinical efficacy of using methotrexate singly or combined with total glucosides of paeony in treating juvenile rheumatoid arthritis," Journal of Taishan Medical College, vol. 32, no. 10, pp. 744-746, 2011 (Chinese).

[32] J. Zhao and H. Li, "Total Glucosides of Paeony combined methotrexate for non-systemic Juvenile Idiopathic Arthritis," World Health Digest Medical Periodieal, vol. 8, no. 46, pp. 8-10, 2011 (Chinese).

[33] P. Fang and L.-H. Li, “The therapeutic effect of total glucosides of paeony combined methotrexate on non-systemic juvenile idiopathic arthritis," Medical Information, vol. 25, no. 1, pp. 168169, 2012 (Chinese).

[34] X.-J. Wang, "Clinical observation of methotrexate combined with total glucosides of paeony for juvenile rheumatoid arthritis," Evaluation and Analysis of Drug-Use in Hospitals of China, vol. 12, no. 8, pp. 722-724, 2012 (Chinese).

[35] J. Higgins, R. Churchill, M. Cumpston, and J. Chandler, Cochrane Handbook for Systematic Reviews of Interventions Version 5.1.0, The Cochrane Collaboration, 2011.

[36] Y.-Q. Zheng, W. Wei, L. Zhu, and J.-X. Liu, "Effects and mechanisms of Paeoniflorin, a bioactive glucoside from paeony root, on adjuvant arthritis in rats," Inflammation Research, vol. 56, no. 5, pp. 182-188, 2007.

[37] L.-L. Zhang, W. Wei, N.-P. Wang et al., "Paeoniflorin suppresses inflammatory mediator production and regulates $\mathrm{G}$ proteincoupled signaling in fibroblast-like synoviocytes of collagen induced arthritic rats," Inflammation Research, vol. 57, no. 8, pp. 388-395, 2008.

[38] X. Li, J. Shen, Z. Zhong, H. Wen, Q. Luo, and W. Wei, "Paeoniflorin: a monomer from traditional Chinese medical herb ameliorates Schistosoma japonicum egg-induced hepatic fibrosis in mice," Journal of Parasitology, vol. 95, no. 6, pp. 15201524, 2009.

[39] X. Li, J. Shen, Z. Zhong et al., "Paeoniflorin ameliorates schistosomiasis liver fibrosis through regulating IL-13 and its signalling molecules in mice," Parasitology, vol. 137, no. 8, pp. 1213-1225, 2010.

[40] D.-F. Liu, W. Wei, and L.-H. Song, "Protective effect of paeoniflorin on immunological liver injury induced by bacillus Calmette-Guerin plus lipopolysaccharide: modulation of tumour necrosis factor- $\alpha$ and interleukin-6 mRNA," Clinical and Experimental Pharmacology and Physiology, vol. 33, no. 4, pp. 332-339, 2006.

[41] H. -C. Li, C. -P. Wen, M. -J. Wang et al., "Meta-analysis of total glucosides paeony combined with methorexate in treating rheumatoid arthritis," Zhong Hua Zhong Yi Yao Za Zhi, vol. 27, no. 4, pp. 1115-1119, 2012 (Chinese).

[42] X.-J. Guo, S. Li, and Y.-S. Huang, "Efficacy and safety of total glucosides of paeony for rheumatoid arthritis: a meta-analysis," Journal of Jinggangshan University, vol. 34, no. 3, pp. 83-87, 2013 (Chinese).

[43] W. Shang, J.-H. Guo, and H. Cai, "Meta-analysis of total glucosides of paeony combined with methotrexate in treatment for rheumatoid arthritis," Modern Journal of Integrated Traditional Chinese and Western Medicine, vol. 19, no. 2, pp. 653-656, 2010 (Chinese). 


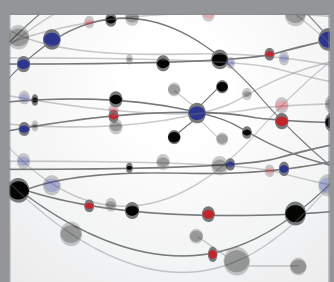

The Scientific World Journal
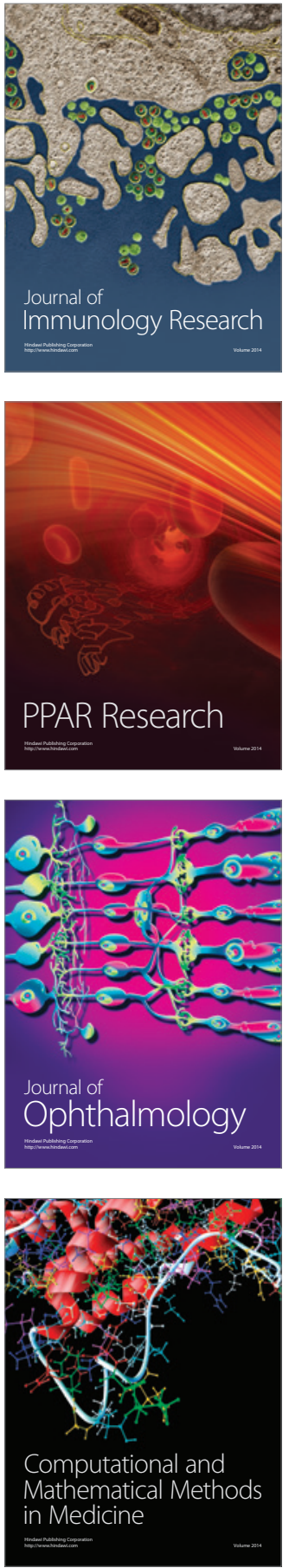

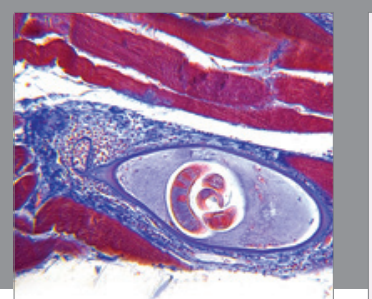

Gastroenterology Research and Practice

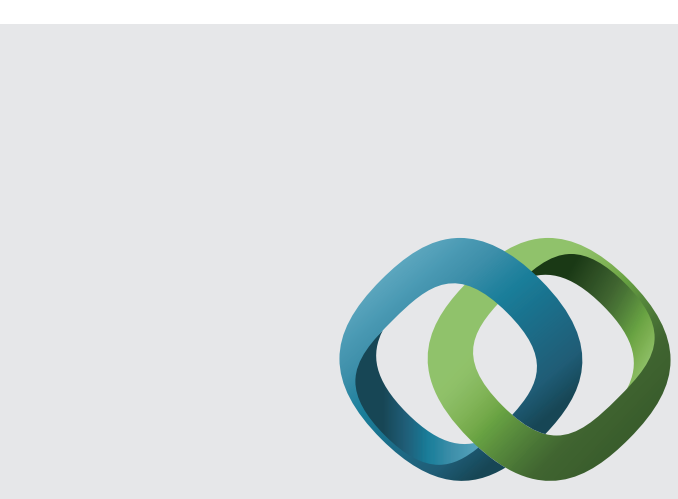

\section{Hindawi}

Submit your manuscripts at

http://www.hindawi.com
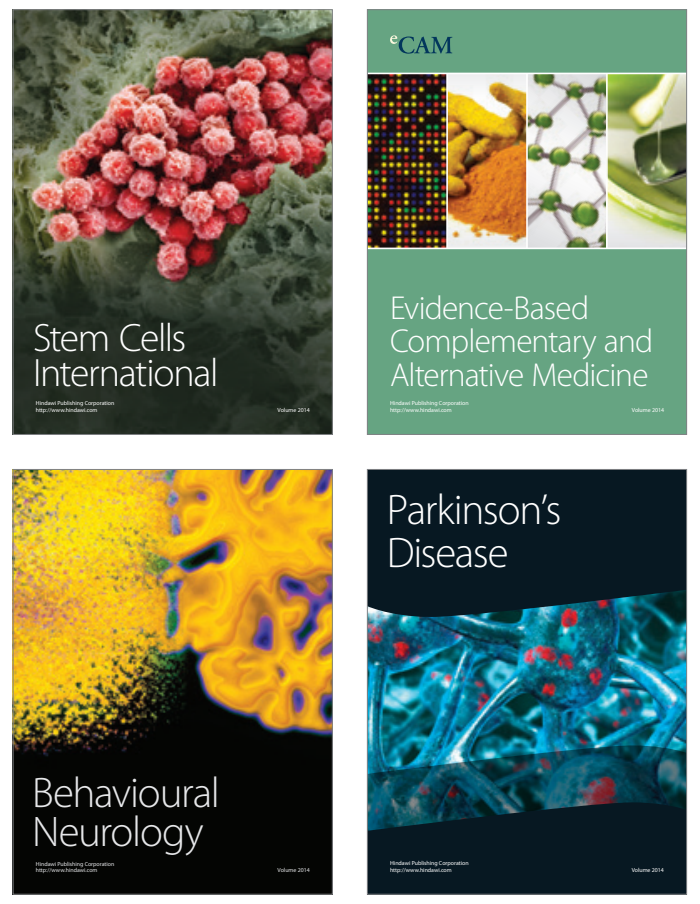
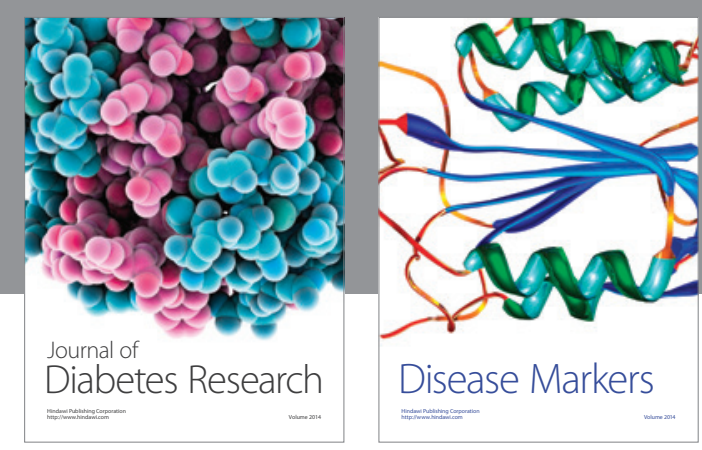

Disease Markers
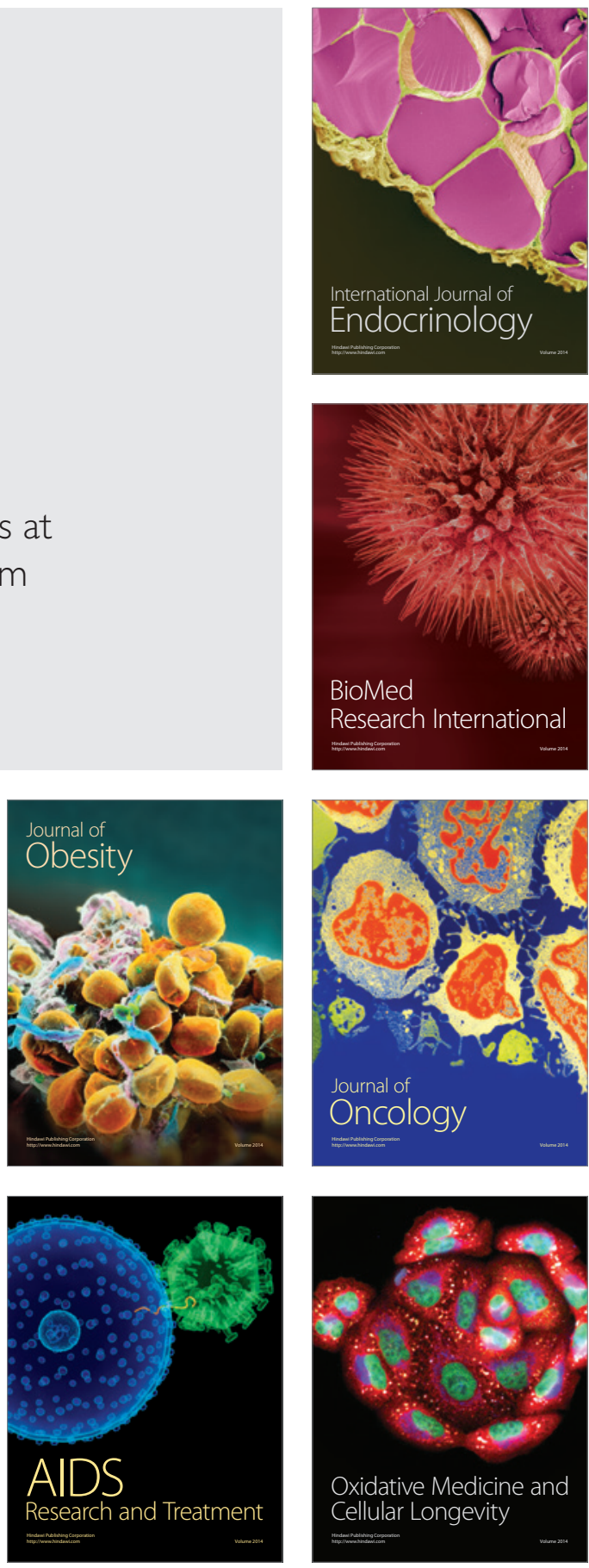